\title{
Mapeamento dos registros de balanço hídrico em uma unidade de terapia intensiva pública no interior sul da Amazônia Legal
}

\author{
Mapping of fluid balance records in a public intensive care unit in the southern region of \\ the Legal Amazon
}

\begin{abstract}
Mapeo de registros de balance hídrico en una unidad de cuidados intensivos pública en el interior sur de la Amazonía Legal
\end{abstract}

Larissa da Silva Gums ${ }^{1 *}$, Jéssica dos Santos Souza ${ }^{1}$, Laurindo Pereira de Souza1.

\section{RESUMO}

Objetivo: Mapear e analisar os registros referentes ao balanço hídrico realizado na unidade de terapia intensiva. Métodos: Trata-se de um estudo descritivo com delineamento transversal e abordagem quantitativa, norteado pela ferramenta Strengthening the Reporting of Observational Studies in Epidemiology (STROBE). Foram analisadas fichas de balanço hídrico referentes aos meses de julho a setembro de 2019. As variáveis numéricas foram submetidas a cálculos de frequência, média e desvio padrão. As variáveis categóricas foram submetidas ao teste de associação Qui-quadrado de Pearson utilizando o programa BioEstat 5.3. Resultados: Foram encontrados registros referentes ao ganho de volume por via oral, enteral e parenteral. No que concerne as perdas, foram encontrados registros de eliminação por diurese, evacuação, vômito, dreno tubular e perdas quantificadas através de hemodiálise. Não foram encontrados registros referentes a perdas insensíveis. Das fichas analisadas, $49 \%$ apresentaram erros de cálculo que ocasionaram em desvios de $10 \mathrm{ml}$ a $2960 \mathrm{ml}$ no resultado do balanço hídrico. A incidência de erros esteve fortemente associada a dois fatores principais: o período do dia e a via. Conclusão: Grande parte dos registros referentes ao balanço hídrico mostraram-se incompletos e possuíam erros de cálculo que podem interferir na avaliação dos pacientes e conseguintemente nas condutas terapêuticas.

Palavras-chave: Equilíbrio hidroeletrolítico, Registros de enfermagem, Unidade de terapia intensiva.

\begin{abstract}
Objective: To map and analyze the records referring to the fluid balance held in the intensive care unit. Methods: This is a descriptive study with transversal design and quantitative approach, guided by the tool: Strengthening the Reporting of Observational Studies in Epidemiology (STROBE). Fluid balance records for the months of July to September 2019 were analyzed. The numerical variables were submitted to frequency calculations, average and standard deviation. The categorical variables were submitted to Pearson's Chisquare association test using the BioEstat 5.3 software. Results: Records relating to oral, enteral and parenteral volume gain were found. With regard to the losses, records of elimination by diuresis, evacuation, vomiting, tubular drain and losses quantified by hemodialysis were found. Records referring to insensitive losses were not found. Of the analyzed records $49 \%$ presented calculation errors that caused deviations of $10 \mathrm{ml}$ to $2960 \mathrm{ml}$ in the fluid balance result. The incidence of errors was strongly associated with two main factors: the time of day and the route. Conclusion: Many records referring to the fluid balance were incomplete and had calculation errors that may interfere in the evaluation of the patients and consequently on the therapeutic conducts.
\end{abstract}

Keywords: Hydroelectrolytic balance, Nursing records, Intensive care unit.

${ }^{1}$ Hospital Regional de Cacoal (HRC), Cacoal - RO. *E-mail: larissagums@gmail.com 


\section{RESUMEN}

Objetivo: Mapear y analizar los registros referentes al balance hídrico realizado en la unidad de cuidados intensivos. Métodos: Se trata de un estudio descriptivo con un diseño transversal y abordaje cuantitativa, orientado por la herramienta Strengthening the Reporting of Observational Studies in Epidemiology (STROBE). Fueron analizadas fichas de los balances hídricos referentes de los meses de julio a septiembre de 2019. Las variables numéricas fueron sometidas a cálculos de frecuencia, media y desvío estándar. Las variables categóricas fueron sometidas a la prueba de asociación Chi-cuadrado de Pearson utilizando el programa BioEstat 5.3. Resultados: Se encontraron registros referentes de ganancia de volumen por vía oral, enteral y parenteral. En lo que se refiere a las pérdidas, se encontraron registros de eliminación por diuresis, evacuación, vómitos, drenaje tubular y pérdidas cuantificadas a través de hemodiálisis. No se encontraron registros referentes de pérdidas insensibles. De las fichas analizadas, $49 \%$ presentaron errores de cálculo que ocasionaron en desvíos de $10 \mathrm{ml}$ a $2960 \mathrm{ml}$ en el resultado del balance hídrico. La incidencia de errores estuvo fuertemente asociada a dos factores principales: el período del día y la vía. Conclusión: Muchos de los registros referentes al balance hídrico estaban incompletos y con errores de cálculo que pueden interferir en la evaluación de los pacientes y consecuentemente en las conductas terapéuticas.

Palabras clave: Equilibrio hidroelectrolítico, Registros de enfermería, Unidad de cuidados intensivos.

\section{INTRODUÇÃO}

O balanço hídrico $(\mathrm{BH})$ consiste na razão entre o volume de líquido recebido e o eliminado pelo organismo. Para sua realização, todos os volumes são medidos e registrados no prontuário do paciente no qual, ao final de um período de $24 \mathrm{~h}$, o volume total de saída deve ser subtraído ao volume total de entrada (KAWAMOTO EE e FORTES JI, 2011). Quando o cálculo do BH resulta em um número positivo, indica que parte dos líquidos administrados não foi eliminada, permanecendo retida no organismo; o contrário acontece com o balanço negativo (CICCIOLI F, 2017).

Durante um dia inteiro, uma pessoa ingere aproximadamente $2,200 \mathrm{ml}$ de água através de comida e bebida, e o metabolismo normal produz em torno de $300 \mathrm{ml}$, o que resulta em cerca de $2,500 \mathrm{ml}$ de água por dia (SILVERTHORN DU, 2016). Mais da metade de todo o líquido ingerido é eliminado pela urina, uma pequena quantidade nas fezes e ainda por outras vias como a pele e os pulmões que são as chamadas de perdas insensíveis, pois não podem ser percebidas de maneira consciente (KAWAMOTO EE, 2018).

Em um indivíduo saudável o volume de líquido eliminado deve ser equivalente ao volume recebido, obtendo um $\mathrm{BH}$ mais próximo de zero. O próprio corpo possui mecanismos de controle e compensação que trabalham para manter esse equilíbrio (CICCIOLI F, 2017). Todavia, em situações patológicas, e principalmente dos pacientes críticos que estão na unidade de terapia intensiva (UTI), algumas alterações como o suor em excesso, febre, diarreia, vômito, queimaduras, entre outras, podem ser grandes fontes de perdas (SILVA GF e COUTO LA, 2007).

Com isso, o monitoramento rigoroso do BH na UTI com o intuito de prever alterações que implicam na condição clínica do paciente torna-se imprescindível. Entretanto, embora seja prática comum da equipe de enfermagem, seus métodos não estão padronizados e podem apresentar resultados imprecisos a depender da forma e dos recursos disponíveis para sua realização (CICCIOLI F, 2017).

O controle da movimentação hídrica no paciente crítico por meio da realização de $\mathrm{BH}$ carrega em si alguns percalços, contudo, ainda é a realidade de muitos hospitais, principalmente aqueles com recursos limitados.

Perren A et al. (2011) investigou fatores relacionados a confiabilidade do BH para a prática clínica e encontrou um resultado insatisfatório quando comparado a medida do peso corporal. Algumas causas foram apontadas como agentes nesse processo, entre elas a inadequação dos métodos de medida e dos registros, sendo este último o cerne do presente estudo. 
A Resolução 564/2017 estabelece como dever dos profissionais de enfermagem "Registrar no prontuário e em outros documentos, as informações inerentes e indispensáveis ao processo de cuidar de forma clara, objetiva, cronológica, legível, completa e sem rasuras" (COFEN, 2017).

A equipe deve estar ciente que para a realização desses registros devem dispor de diligência, a fim de garantir anotações reais de todos os líquidos, assegurando resultados confiáveis (OLIVEIRA SKPD, et al. 2010). A precariedade dos registros pode afetar a qualidade da assistência acarretando riscos ao paciente (KITA VY e HASEGAWA SM, 2020).

Em face das considerações supracitadas e considerando que para garantir uma assistência segura, eficaz e de qualidade é fundamental a implementação de práticas baseadas em evidencias (PBE) de maneira que atenda às necessidades do doente melhorando seu desfecho clínico, o objetivo desta pesquisa foi mapear e analisar os registros referentes ao balanço hídrico realizado na unidade de terapia intensiva.

\section{MÉTODOS}

Trata-se de um estudo descritivo com delineamento transversal e abordagem quantitativa, norteado pela ferramenta Strengthening the Reporting of Observational Studies in Epidemiology (STROBE). O estudo foi realizado na UTI adulto de um hospital público de referência em atendimento de nível secundário e terciário no interior sul do estado de Rondônia.

A população estudada foram as fichas de balanço hídrico realizadas na UTI no período de 10 de julho a 30 de setembro de 2019. A seleção se deu a partir dos registros do livro de admissão da unidade.

Os critérios de inclusão foram registros de informações superiores a $24 \mathrm{~h}$, com recorte limite no sétimo dia de internação definido com base no tempo médio de internação da instituição. Os critérios de exclusão foram fichas referentes a pacientes em pós-operatório que receberam alta ou evoluíram para óbito antes de 48 horas completas da admissão, e fichas com grafia ilegível.

Foram identificadas 324 fichas, 37 não atendiam ao critério de seleção e 87 não foram localizadas no Serviço de Arquivo Médico (SAME). Diante dos critérios supracitados selecionou-se 200 fichas de balanço hídrico $(\mathrm{BH})$ de $24 \mathrm{~h}$ completas.

O instrumento utilizado para coletar as informações foi construído pelos próprios pesquisadores com base na literatura pertinente e experiência adquirida da prática assistencial. Esse instrumento foi aplicado como "teste piloto" em 5 amostras. As lacunas existentes foram corrigidas e validadas e oficialmente estruturado em planilha eletrônica composta por campos destinados a anotações pertinentes a todo volume recebido e eliminado pelo paciente.

A respeito dos volumes recebidos, foram analisados aqueles administrados por via oral, enteral e parenteral. Quanto aos volumes eliminados, foram considerados os relacionados a diurese, evacuação, vômito, sangramento, drenagens, perdas quantificadas através de hemodiálise e perdas insensíveis.

Após levantamento e tabulação dos dados, foram recalculados todos os balanços com auxílio da ferramenta automática de cálculo do programa MS Exce ${ }^{\circledR}$. A classificação de erro de cálculo foi considerada quando o resultado do recálculo feito pelos autores fosse diferente.

Depois da sistematização dos dados, foi realizada análise das variáveis numéricas a partir de cálculos de frequência, média e desvio padrão no programa MS Exce ${ }^{\circledR}$. As variáveis categóricas foram submetidas ao teste de associação Qui-quadrado de Pearson e foi considerado como resultado estatisticamente significativo um valor $p<0,05$. As tabelas $2 \times 2$ receberam correção de Yates. $O$ programa utilizado foi 0 BioEstat 5.3.

O projeto foi aprovado pelo Comitê de Ética e Pesquisa (CEP) com parecer n³.718.259. 


\section{RESULTADOS}

Foram analisadas 200 fichas de balanço hídrico, destas, 98 (49\%) apresentaram erros de cálculo. Os resultados das variáveis analisadas estão descritos nas Tabelas 1 e 2.

As diferenças encontradas entre o resultado do $\mathrm{BH}$ registrado nas fichas e o obtido no recálculo realizado pelos pesquisadores foi no mínimo $10 \mathrm{ml}$ e no máximo $2960 \mathrm{ml}$, sendo que a maioria se concentrou entre $\leq 100 \mathrm{ml}(53.06 \%)$ e $101-500 \mathrm{ml}(33,67 \%)$ (Tabela 1).

Tabela 1 - Distribuição das diferenças de volume (ml) encontradas no resultado dos balanços hídricos em decorrência dos erros de cálculo.

\begin{tabular}{lllllll}
\hline & \multicolumn{7}{c}{ Volume (ml) } \\
\cline { 2 - 7 } & $\mathbf{5 1 0 0}$ & $\mathbf{1 0 1 - 5 0 0}$ & $\mathbf{5 0 1 - 1 0 0 0}$ & $\mathbf{1 0 0 1 - 1 5 0 0}$ & $\mathbf{1 5 0 1 - 3 0 0 0}$ & Total \\
\hline $\mathbf{N}$ & 52 & 33 & 6 & 5 & 2 & 98 \\
\hline$\%$ & 53.06 & 33.67 & 6.12 & 5.10 & 2.04 & 100 \\
\hline Média & 46 & 279 & 662 & 1194 & 2609 & 273 \\
\hline Desvio padrão & 33.03 & 134.04 & 125.05 & 178.19 & 496.39 & 451.99 \\
\hline Intervalo & $10-100$ & $104-500$ & $540-839$ & $1069-1500$ & $2258-2960$ & $10-2960$ \\
\hline
\end{tabular}

Fonte: Gums LS, et al. 2020.

Os dados revelam ainda que em $70.41 \%$ das fichas com erros de cálculo, o resultado registrado era menor que o obtido pelos pesquisadores no recalculo. A maioria dos erros estava relacionada ao volume recebido pelo paciente (80.61\%) enquanto apenas $12,24 \%$ às vias de eliminações. Em relação ao período do dia, houve uma predominância do período noturno $(58,16 \%)$ em comparação ao período diurno (30.61\%). A maior parte das fichas apresentou apenas um erro de cálculo (75.51\%), porém, foram encontradas ficham que continham mais de três erros (7.14\%) (Tabela 2).

Tabela 2 - Análise descritiva das fichas de balanço hídrico com erros de cálculo segundo características da interferência no resultado, via, período do dia, e quantidade de erros.

\begin{tabular}{ccll}
\hline Variável & $\mathbf{n}$ & $\%$ & $\mathbf{p}^{\mathbf{*}}$ \\
\hline Resultado & & & \\
\hline Maior & 29 & 29.59 & $<0,0001$ \\
Menor & 69 & 70.41 & $<0,0001$ \\
\hline Via & & & \\
\hline Entrada & 79 & 80.61 & \\
Eliminação & 12 & 12.24 & \\
Ambas & 7 & 7.14 & $<0,0001$ \\
\hline Período & & & \\
\hline Diurno & 30 & 30.61 & \\
Noturno & 57 & 58.16 & \\
Ambos & 11 & 11.22 & \\
\hline Quantidade de erros & & & \\
\hline 01 & 74 & 75.51 & \\
02 & 17 & 17.35 & \\
$\geq 03$ & 7 & 7.14 & \\
\hline
\end{tabular}

Fonte: Gums LS, et al. 2020.

Os registros encontrados são pertinentes ao ganho de volume por via oral e enteral (dieta e medicação) e via parenteral (fluidoterapia, hemotransfusão, medicação, sedação e drogas vasoativas). No que concerne as perdas, foram encontrados registros de eliminação por diurese, evacuação, vômito, dreno tubular e perdas quantificadas através de hemodiálise.

Não foram encontrados registros referentes a perdas insensíveis. Algumas fichas constavam o registro de evacuação pelo sistema de cruzes (+), entretanto, a instituição não possui padronização para cálculo da equivalência do símbolo. 


\section{DISCUSSÃO}

Os erros de cálculo podem interferir no resultado do BH e torná-lo impreciso no apontamento do saldo de volume de líquido ao final do período. Diacon A e Bell J (2014) analisaram os registros das primeiras 48h de internação em 103 prontuários e evidenciaram uma diferença de até $500 \mathrm{ml}$ em 68,9\% dos prontuários analisados, semelhante ao descrito no presente estudo.

Apesar da maioria das fichas analisadas neste estudo apresentarem um desvio de até $500 \mathrm{ml}$ no resultado do $\mathrm{BH}$, não se pode ignorar o fato de que $7 \%$ apresentaram diferenças em um intervalo de $1.069 \mathrm{ml}$ a $2.960 \mathrm{ml}$, entregando um registro de balanço completamente discrepante da real condição do paciente.

Outros aspectos relacionados a qualidade do registro podem levar a um resultado de BH equivocado, como descrito por Asfour $\mathrm{HI}$ (2016), que realizou uma auditoria dos volumes que eram considerados pelos enfermeiros de uma UTI para o cálculo de equilíbrio de líquidos e verificou que $35 \%$ não abrangiam a completude de volumes recebidos e eliminados pelos pacientes. Dentre esses, alguns balanços registravam um volume médio de $467,65 \mathrm{ml}$ acima do real e de $337,78 \mathrm{ml}$ quando abaixo.

No presente estudo, $70,41 \%$ dos resultados registrados eram menores que o identificado pelos pesquisadores no recalculo, o que significa que por 69 vezes, os pacientes fizeram um balanço mais positivo do que fora registrado no prontuário.

O paciente crítico subordinado a sobrecarga hídrica está sujeito a complicações clínicas que favorecem a morbimortalidade dentre as quais se destacam: congestão pulmonar com piora na complacência e troca gasosas, edema miocárdico com distúrbio de condução e disfunção diastólica, além de edema cerebral, renal e de tecidos periféricos que podem comprometer a cicatrização de feridas (ÁVILA MON, et al., 2014).

Há muito, o BH vem sendo investigado como preditor de desfechos clínicos, com potencial para ser amplamente utilizado na assistência aos pacientes críticos, fortemente associado principalmente a mortalidade e sobrevida na sepse e choque séptico (TIGABU BM, et al., 2018; DHONDUP T, et al., 2018; NEYRA JA, et al., 2016) e afecções renais (ÁVILA MON, et al., 2014, TEIXEIRA C, et al., 2013; PAYEN D, et al., 2008).

Alsous F, et al. (2000), realizaram um pequeno estudo piloto no qual encontrou uma associação significativa na redução da mortalidade nos pacientes que apresentaram um balanço negativo $>500 \mathrm{ml}$ em mais de um dos 03 primeiros dias do choque séptico.

Dado isso, reforça-se a importância do cálculo correto do balanço hídrico, pois os erros nos registros podem prejudicar a avaliação do paciente e consequentemente a conduta terapêutica, colocando em risco sua saúde e segurança, principalmente naqueles pacientes que necessitam de um controle rigoroso e ao considerar a avaliação do balanço hídrico acumulado.

Diacon A e Bell J (2014) constatou que dentre os pacientes que tiveram sua ficha de BH calculada de forma imprecisa, $38,8 \%$ receberam administração de diuréticos e ressalta que, considerando isso, a terapia pode ter sido aplicada de maneira equivocada e resultar em desequilíbrio hídrico que afeta a estabilidade hemodinâmica.

Dentre as fichas analisadas, houve um predomínio da ocorrência de erro nos cálculos pertinentes aos volumes recebidos pelo paciente $(80,61 \%)$. Melo EM, et al. (2014) ao fazerem avaliações dos registros de enfermagem, observou que a maioria dos valores das soluções infundidas tinha sua soma correta $(90,20 \%)$, contudo o desempenho foi ainda superior na soma das eliminações com $94,10 \%$ de cálculos corretos. É visível que há instituições em que os erros são menos frequentes, porém, esse resultado reforça o que foi encontrado a despeito da periodicidade de erros atrelados às anotações do ganho de volume.

Uma hipótese para justificar esse achado, é a existência de maior número de lançamentos nesta sessão em comparação a sessão de eliminações. Essa diferença no número de lançamentos é comum ao perfil dos pacientes de UTI que, com frequência, estão recebendo sedativos, drogas vasoativas e fluidos por meio de bombas de infusão contínua (BIC), além de medicamentos da prescrição médica e dieta, enquanto eliminação por vias além de diurese e evacuação se aplicam a casos mais específicos. 
Netto SM, et al. (2015) compararam os registros de um grupo de pacientes que possuíam apenas um sistema de drenagem com outro grupo em que os pacientes possuíam dois, três ou quatro sistemas de drenagens e encontraram $94,1 \%$ de descrições corretas no primeiro grupo contra $27,8 \%$ no segundo. Observaram que a presença de mais de um sistema de drenagem esteve associada a não especificação correta dos volumes.

Houve também uma associação estatisticamente significativa $(p<0,0001)$ entre a ocorrência de erros de cálculo e o período de trabalho, sendo o período noturno o que mais apareceu. $O$ trabalho no período noturno já vem sendo mencionado com fator de risco para eventos adversos pois afeta diretamente no padrão sono-vigília, visto que o sono é considerado uma necessidade humana básica. Essa privação reflete no estado de alerta e atenção, debilitando a segurança e a prática da assistência (SILVA APD, et al. 2017).

Além disso, na unidade estudada, é rotina o revezamento do horário de repouso no período noturno, fazendo com que o cálculo do $\mathrm{BH}$ nesse período seja realizado por uma equipe reduzida. Muito se é discutido, sobre o dimensionamento de enfermagem no processo de gestão em saúde. Quando não realizado de forma adequada à demanda do setor, pode prejudicar a prestação de cuidados, influenciando tanto em resultados clínicos como em alterações de prognósticos. Ou seja, o dimensionamento de pessoal da enfermagem é visto como critério na qualidade da assistência (QUEIJO AF, et al., 2020).

Sobre os lançamentos encontrados registrados com símbolo (+), foi observado um padrão de cálculo onde foi considerado o valor de $25-50 \mathrm{ml}$ para cada símbolo registrado, levando a uma variação no volume a depender de dois fatores, primeiro do profissional que avaliou o fluido perdido e realizou o lançamento, e posteriormente, do entendimento do enfermeiro que fez o cálculo.

Todavia, apesar do enfermeiro demonstrar capacidade de estimar volumes com certa precisão, é indispensável que a mensuração de entradas e saídas sejam de fato medidas e não apenas estimadas (DAFFURN K, et al. 1994, NETTO SM, et al. 2015). O registro de perda de volume pelo sistema de cruzes (+) não proporciona medida exata do volume perdido, além disso a ausência de protocolo institucional que estabeleça o valor equivalente em ml dificulta ainda mais a precisão do cálculo.

Apesar das vias de eliminação gastrointestinal e vesical serem as mais comuns relacionado a registros de perdas, não são as únicas que podemos considerar perdas de líquidos. Existem ainda as perdas insensíveis, as quais não são mensuráveis com clareza, e que ocorrem através da pele, respiração, sudorese, febre, evacuação e diarreia (KITA VY e HASEGAWA SM, 2020).

Para essas vias, portanto, a maneira de inseri-las no $\mathrm{BH}$ se dá ao estimá-las por meio de referências e fórmulas previamente estabelecidas na literatura. Um exemplo seria na perda de água por febre, sendo para cada grau acima de $37,8^{\circ} \mathrm{C}$ a ocorrência da eliminação de $100 \mathrm{ml} / \mathrm{kg}$ de água (KITA VY e HASEGAWA SM, 2020).

No entanto, a aplicação desses métodos é questionável, pois apesar de levar a um resultado mais próximo do obtido através da pesagem dos pacientes comparados, ainda se mantém muito distante de uma concordância com o mesmo. Além disso, algumas medidas parecerem aplicáveis apenas a tipos específicos de subpopulação (PERREN A, et al., 2011).

Estudos hodiernos revelam que nenhuma fórmula de realização de $\mathrm{BH}$ encontrou bom nível de concordância com o monitoramento do peso corporal, porém a pesagem diária do paciente internado em UTI torna-se inviável pela condição crítica do doente (GUPTA V e DESHPANDE K, 2019). Uma alternativa são as camas com balança integrada, entretanto, essa realidade pode estar longe de ser alcançada em hospitais públicos brasileiros.

É concludente que a busca por métodos que demonstrem maior precisão em monitorar a movimentação de líquidos em pacientes críticos deve ser continuada, contudo, mudanças básicas como o correto registro das informações devem receber mais atenção para que garanta a efetividade do procedimento.

Netto SM, et al. (2015) dizem que os profissionais responsáveis pela execução das atividades assistenciais estão mais envolvidos com a rotina emanada do setor, considerando como menos importante a efetivação de registros. Essa perspectiva deve ser discutida, pois a garantia de uma assistência de qualidade dentro da terapia intensiva tem total relação com a realização dessa ação. 
As informações inerentes ao processo de cuidados dos pacientes, por vezes, são incompletas ou ausentes, contrariando o que é estabelecido nos códigos de éticas profissionais (GOMES LEM, et al., 2020).

As ações de enfermagem voltadas ao cuidado exigem dedicação do profissional para garantia de qualidade na assistência. Nesse contexto a adoção de atitudes incoerentes com o que é exigido da profissão é considerada uma imperícia, imprudência e até mesmo negligencia (CARBONI RM, REPPETTO MA, NOGUEIRA VDO, 2018).

A falta de compreensão sobre as atribuições de cada profissional, associado a carência de conhecimento sobre a importância clínica do registro do balanço hídrico, podem levar o enfermeiro a se tornar omisso neste processo, favorecendo a ocorrência de erros (MARQUES SF DA S, et al. 2011).

Destarte, é imperativo ao enfermeiro o gerenciamento de sua equipe, uma vez que é responsável por supervisionar e avaliar os registros do BH (SOUZA JDS, et al., 2020). Logo, o enfermeiro não pode ignorar o oficio de provedor das práticas cientificas que regem a excelência da assistência .

Por tratar-se de uma atividade exercida por seres humanos, a ocorrência de falhas no BH pode ser considerada inerente a sua forma de realização não automatizada, contudo, não se pode ter isso como hábito, principalmente na terapia intensiva, é imprescindível saber reconhecer as falhas que comprometem a assistência. Por isso ressalta-se aqui a necessidade de educação continuada a fim de evitar os erros preveníveis (FORTE, et al., 2017).

Algumas maneiras sugestivas de sistematizar esses registros e melhorar a qualidade da assistência seriam a padronização de um funcionário responsável por lançar os valores e calcular o $\mathrm{BH}$; registrar também as perdas insensíveis através de protocolos com embasamento científico; lançar na folha de registro o item "balanço hídrico acumulado" e implantar na unidade de terapia intensiva registros eletrônicos (BARCELOS DGD e CRUZ ICFD, 2016).

De forma geral, há muito o que ajustar no monitoramento de líquidos no paciente crítico, tanto na padronização dos métodos de mensuração e cálculo, quanto na qualidade do registro propriamente dito. $O$ presente estudo teve a intenção de expor parte do resultado de uma pesquisa, e teve como limitação a escassez de estudos com abordagem semelhante sobre a temática, dificultando a comparação dos resultados.

\section{CONCLUSÃO}

O balanço hídrico realizado através de anotações manuais dos ganhos e perdas do paciente crítico se mostrou passível de erros, tanto pela ausência de lançamento de volumes de entrada e de saída, como pelo cálculo incorreto dos volumes lançados. Estas falhas podem ser potencializas a depender do nível de conhecimento dos profissionais, além de fatores extrínsecos como o tipo de lançamento e o turno de trabalho. Tendo em vista esse desfecho, é necessário dedicar maior atenção à realização do balanço hídrico para que se reduzam a ocorrência de erros, além de envidar esforços na busca de métodos mais precisos de mensuração que possam colaborar para uma melhor assistência de saúde no ambiente de terapia intensiva.

\section{REFERÊNCIAS}

1. ALSOUS F. et al. Negative fluid balance predicts survival in patients with septic shock: A retrospective pilot study. Chest, 2000; 117(6): 1749-1754.

2. ASFOUR HI. Fluid Balance Monitoring Accuracy in Intensive Care Units. IOSR J Nurs Heal Sci, 2016; 05(04): 53-62.

3. ÁVILA MON, et al. Balanço hídrico, injúria renal aguda e mortalidade de pacientes em unidade de terapia intensiva. Jornal brasileiro de nefrologia, 2014; 36(3): 379-388.

4. BARCELOS DGD, CRUZ ICFD. Water balance: systematized literature review for a clinical protocol. Journal of Specialized Nursing Care, 2016; 8(1): 1-5.

5. COFEN. Resolução $n^{\circ} 564 / 2017$. Aprova o novo código de ética dos profissionais de enfermagem. Conselho Federal De Enfermagem. Brasília (DF): COFEN, 2017.

6. CARBONI RM, et al. Erros no exercício da enfermagem que caracterizam imperícia, imprudência e negligência: uma revisão bibliográfica. Rev Paul Enferm, 2018; 29:100-107. 
7. CICCIOLI F. O manejo do balaço hídrico. In: VIANA, RAPP, TORRE, M. Enfermagem em Terapia Intensiva: Práticas integrativas. Barueri, SP: Manole, 2017; 356-366 p.

8. DAFFURN K, et al. Fluid balance charts: do they measure up?. Br J Nurs, 1994; 3(16): 816-20.

9. DIACON A, BELL J. Investigating the recording and accuracy of fluid balance monitoring in critically ill patients. Southern African Journal of Critical Care, 2014; 30(2): 55-57.

10. DHONDUP T. et al. Association of negative fluid balance during the de-escalation phase of sepsis management with mortality: A cohort study. Journal of Critical Care, 2018; 55: 16-21.

11. FORTE ECN, et al. Erros de enfermagem: o que está em estudo. Texto \& Contexto-Enfermagem, 2017; $26: 22-30$.

12. GOMES LEM, et al. O prontuário do paciente e o dever legal e ético de registro dos profissionais da saúde: uma revisão literária. Revista Eletrônica Acervo Saúde, 2020; 12 (7): e3815.

13. GUPTA V, DESHPANDE K. Relationship between body weight and fluid balance in critically ill patients - A prospective observational study. Journal of Critical Care, 2019; 53: 244-247.

14. KAWAMOTO EE, FORTES JI. Fundamentos de Enfermagem. Rio de Janeiro: Editora Guanabara Koogan, 2011; $101 \mathrm{p}$.

15. KAWAMOTO EE. Anatomia e fisiologia para enfermagem. Rio de Janeiro: Editora Guanabara Koogan, 2018; 16p.

16. KITA VY, HASEGAWA SM. Balanço hídrico: importância e precisão. In: VIANA RAPP, WHITAKER IY, ZANEI SSV. Enfermagem em Terapia Intensiva Práticas e Vivências. Porto Alegre: Artmed, 2020; 130-135p.

17. MARQUES SFS, et al. Balanço hídrico em recém-nascidos com extremo baixo peso: o conhecimento dos profissionais de enfermagem. Com Ciências Saúde, 2011; 22(1): 41-50.

18. MELO EM, et al. Avaliação dos registros de enfermagem no balanço hídrico de pacientes em unidade de terapia intensiva. Rev enferm UFPI, 2014; 3(4): 35-41.

19. NETTO SM, et al. Análise dos registros referentes ao balanço hídrico em unidade de terapia intensiva. Revista de Enfermagem, 2015; 9(1): 448-456.

20. NEYRA JA, et al. Cumulative Fluid Balance and Mortality in Septic Patients with or Without Acute Kidney Injury and Chronic Kidney Disease. Critical Care Medicine, 2016; 44(10): 1891-1900.

21. OLIVEIRA SKPD, et al. Balanço Hídrico Na Prática Clínica De Enfermagem Em Unidade Coronariana. Revista da Rede de Enfermagem do Nordeste, 2010; 11(2): 112-120.

22. PAYEN D, et al. A positive fluid balance is associated with a worse outcome in patients with acute renal failure. Critical Care, 2008; 12(3): 1-7.

23. PERREN A, et al. Fluid balance in critically ill patients should we really rely on it? Minerva Anestesiologica, 2011; 77(8): 802-811.

24. QUEIJO AF, et al. Mensuração da carga de trabalho de enfermagem na UTI. In: VIANA RAPP, WHITAKER IY, ZANEI SSV. Enfermagem em Terapia Intensiva Práticas e Vivências. Porto Alegre: Artmed, 2020; 39-49.

25. SILVA APD, et al. Trabalho Noturno Na Vida Dos Enfermeiros. Revista Enfermagem Contemporânea, 2017; 6(2): 177.

26. SILVA GF, COUTO LA. Exame clínico do sistema renal. Palomo JSH. Enfermagem em cardiologia: cuidados avançados. Barueri: Manole, 2007; 297.

27. SILVERTHORN DU. Fisiologia humana: uma abordagem integrada. 7nd ed. Porto Alegre: Artmed, 2017; 620p.

28. SOUZA JDS, et al. Food Botulism: Nursing Actions to the Patient that Evolved with Acute Kidney Insufficiency in the Intensive Care Unit. International Journal of Advanced Engineering Research and Science, 2020; 7(6): 207-213.

29. TEIXEIRA C. et al. Fluid balance and urine volume are independent predictors of mortality in acute kidney injury. Critical Care, 2013; 17(1): 1-11.

30. TIGABU BM, et al. Fluid volume, fluid balance and patient outcome in severe sepsis and septic shock: A systematic review. Journal of Critical Care, 2018; (48): 153-159. 